\title{
Development \& Implementation of Smart Vehicle Over Speeding Detector using IoT
}

\author{
Mohammad Ahmar Khan ${ }^{1}$, Sarfraz Fayaz Khan ${ }^{2}$, Taher Alawi Taher Barham BaOmar ${ }^{1}$, Abdul Rahman Mohammed Aqeel Ba Omar \\ ${ }^{1}$ Department of Management Information System, College of Commerce and Business Administration, Dhofar University, 211, \\ Salalah, Sultanate of Oman \\ ${ }^{2}$ Department of Information \& Communication Technology, Algonquin College, K2G1V, Ottawa, Canada
}

\begin{tabular}{l} 
A R T I C L E I N F O \\
\hline Article history: \\
Received: 06 January, 2019 \\
Accepted: 28 February, 2019 \\
Online: 25 March, 2019
\end{tabular}

Keywords:

Over speeding

Internet of Thing

Vehicle Over Speed Detection

Smart Vehicle

\begin{abstract}
A B S T R A C T
Nowadays over speeding is one of the most common traffic violations. Generally, over speeding is the result of restless and bad behavior of drivers. As the accident rates are increasing it is important to develop and implement a system which can automatically detect and report over speeding to the traffic control authorities as early as possible. Nearly all the roads are marked with speed limits depending upon the size of moving vehicles and heaviness of traffic, but some drivers habitually ignore this speed limit. The advancement in technology has replaced most of the manual or semi-automatic systems with an automated system. To add on to various systems in place, this research is making the use of Internet of Things to detect and report over speeding of the vehicle on which the device has been preinstalled. IoT is a technique to integrate various devices to exchange data among themselves. This research proposes the design, development and functioning of a smart device that helps in automatically detect and report to competitive authority, when so ever the subject vehicle exceeds the speed limit. The device has been developed based on the Global Positioning System (GPS) Technology using Raspberry Pi hardware and Android OS and has been practically tested on real time basis by installing it in a car.
\end{abstract}

\section{Introduction}

One of the main causes for accidents all over the world is rash driving. The traffic has increased in many folds but the system of monitoring the speed has been compromised. Rash driving causes severe risk not only to the driver \& passenger but also to general public. Despite the fact that rash driving is a serious issue its present detection methods by patrol officers lacks efficiency [1].

Speed increase multiplies the hazard of accident and the risk of injury during the accidents [2]. At greater speed the vehicle needs a larger distance to stop. The need of hour is to develop a system that will detect and report the over speed of vehicles. The other factors like conditions of weather and driving at night also impact the manual system.

Daily death toll of India due to road accidents is greater than 4 times the yearly death toll from terrorism [3]. 1,39,671, people lost their lives on roads of India during 2014 that is 382 deaths every day. If 57,844 people lost their lives due to over speed nearly 6,969

*Dr. Mohammad Ahmar Khan, Dept. of MIS, CCBA, Dhofar University, PO Box 2509, PC 211, Sultanate of Oman+96898188243 mkhan@du.edu.om
Indians died because of speed driving post consumption of alcohol in 2014. The below figure shows the deaths caused due to accidents in roads

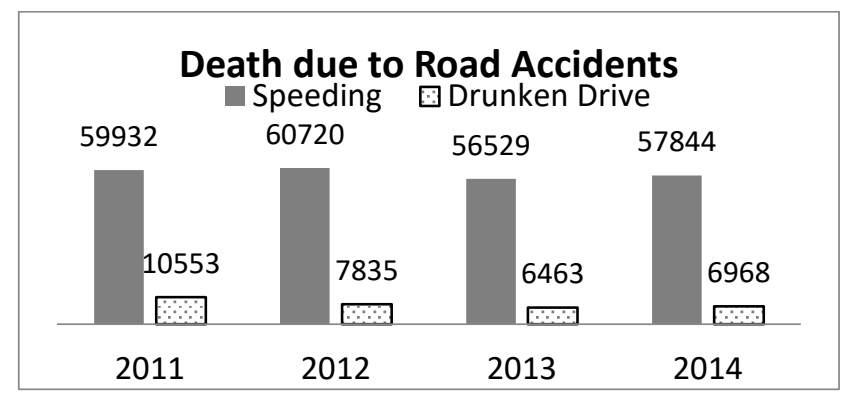

Figure 1: Deaths caused due to accidents in roads Source: [3]

From the above figure it's evident that over speeding is the major cause of death on road accidents from 2011 to 2014. Over speed was responsible for 41 percent of deaths in accidents while dangerous or careless driving claimed 32 percent of deaths in accidents [4]. Mechanical defects in vehicles and bad weather conditions are other causes for deaths in accidents. Maharashtra and Tamil Nadu has reported several numbers of deaths due to over 
speed of vehicles. The below figure shows the fatal road accidents by causes in 2015

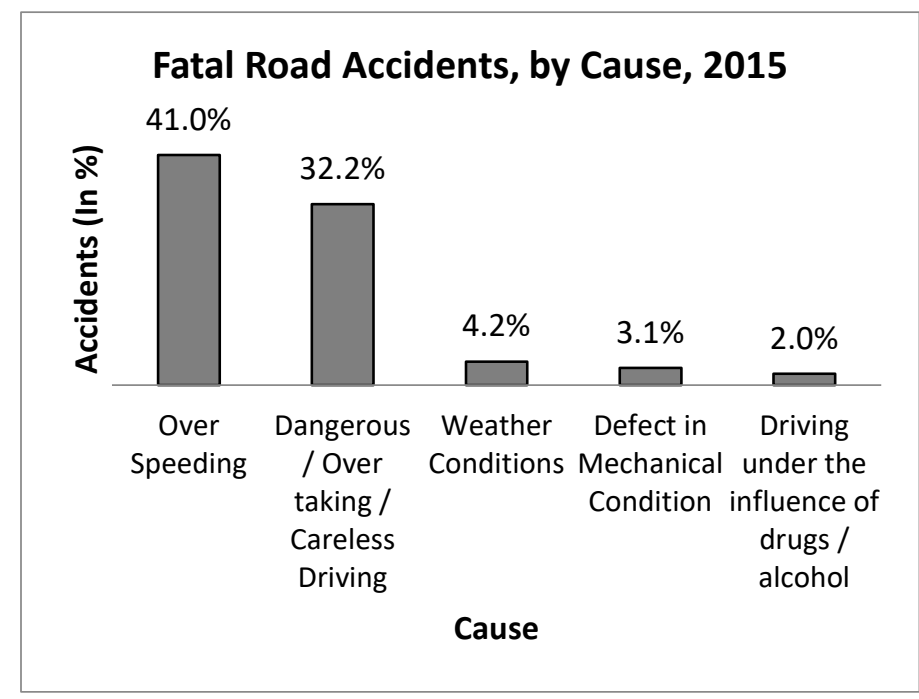

Figure 2: Fatal Road BY Cause in 2015

Source: National Crime Boards Bureau

In [5] the author has mentioned that a vehicle travelling on greater speed will have greater influence during the accident and may cause more number of injuries. Within the driver's fault category during 2016 accidents caused and killed individuals due to over speed accounted for greater number of 2,68,342 out of $4,03,599$ accidents and 73,897 deaths out of 1,21,126 deaths respectively.

The author in [6] has stated that the over speed issue necessitates to develop a new way for managing the traffic system to avoid road accidents. This can be handled through real time traffic management using internet of things. Internet of things is a network which handles the physical devices through internet and this network offers rapid, exact and precise results. Internet of things system is designed to store entire database in PC and this storage is performed mainly through internet. Further this database is used accordingly as their applications and needs.

In [7] the author has mentioned that Internet of things system enhances the components to be accessed from far distanced which mainly reduces the work of humans or their interferences and this makes it much economical. Internet of things is linking various devices of application to each other through internet and this is possible because of sensors which support to transmit vast number of data location.

Internet of things connects the real world objects to virtual globe [8]. It comprises to a globe where living beings and physical objects as well as virtual environments and data, communicate with each other. Internet of Things based intelligent transportation systems are designed to assist the vision of Smart city which targets at using the powerful and advanced communications techniques for the administration of the citizens and the city. Internet of Things plays an essential part in the management of traffic enhancing the effectiveness of data transmission, developing the conditions of traffic, efficiency of management, reducing the costs of management and traffic safety.

Internet of Things is a global network linking the entire smart objects together [9]. The benefits of using internet of things in managing traffic are control of traffic, detection of human proximity, avoidance of theft, emergency response, avoidance of accident and autonomous vehicles. Similarly, the drawbacks of internet of things in managing traffic are network failure and security.

In [10] the authors had stated that enabling internet in every vehicle on the road can pave way for whole vehicle automation and traffic. The internet of things concept can be expanded to entire transport modes making an essential difference in the way that interaction exists between various modes of transport.

Thus it can be inferred that internet of things is an effective way for management of traffic and makes the road travel good for everybody in future.

\section{Literature Review}

The present modern human civilization era the traffic medium plays an essential part in the economic growth of the country [11]. Nowadays a control system is capable to manage such circumstances but not that much efficiently because it needs feedback from humans. A system is needed which must provide continuous update of satellite so that it can manage the traffic smoothly. This will be carried out using IoT using the transmission control protocol/internet protocol. A centralized server must be framed which many of the humans will view after entire analysis of traffic. Relying upon the traffic intensity the diversion path and waiting time will be determined.

In [12] the authors had presented a traffic and vehicle monitoring system based on Internet of Things. Traffic in urban regions and modern cities is making huge risk and is a main concern for the administration and public system. The circumstances namely traffic jams and accidents have become similar because of exponential development in vehicles on road. While human mistakes are one of the major reasons for these issues and lack of appropriate steps and adaptive control system of traffic is another reason. Security for vehicles is also essential. Even in this latent technical globe hackers are handling to break the aspects of security incorporated in modern vehicles. Several technologies namely Bluetooth, Radio Frequency Identification, GSM-GPS and Zigbee based systems were developed but they have restrictions in terms of usage and operation. IOT is a technology that links different objects and is developing at a rapid rate. This system is capable of resolving issues such as congestion in traffic, spotting of vehicle, early warnings considering traffic jams, emergency and VIP clearance of vehicles.

In [13], the researcher presented in their research that urban mobility is one of the unprecedented barriers to be handled in the management of a large city in the contemporary world. This research examines the every developing Urban population around the world and explains about the traffic systems in densely populated cities. Further an advanced system of traffic management is proposed and implemented using IoT (internet of things). The system is assisted by a circuit embedded in the vehicle which runs using radio frequency identification with clustered systems. The system functionalities involves effective light control of traffic, identification of parking space and security mechanism 


\section{M.A. Khan et al. / Advances in Science, Technology and Engineering Systems Journal Vol. 4, No. 2, 170-175 (2019)}

of antitheft. The suggested architecture and process with big data analytics including Hadoop is presented and supervised learning methods are suggested that would support in deciding the roads standard, evaluating overall flow of traffic and examining the vehicles traffic path.

The authors in [14] proposed an intelligent traffic management based on internet of things. The congestion of traffic is a big problem in several big cities of India and the lights of traffic are use basically to manage the vehicle flow. Signal failure, huge number of vehicles, bad enforcement of law and poor management of traffic results in congestion of traffic. One of the main problems with the cities in India is that the existing infrastructure is not expanded much so there have only one choice available which manages traffic better. The traffic control system effectiveness relies on its capability to react on conditions of real time traffic. However conventional system of traffic control is not capable to perform this whatever the density of traffic low or high the signals are timed and performed according to that time. This result in increased congestion in traffic along the roads which outcome in pollution of air. This research proposed a smart traffic management system based on IoT to assist the traffic officers decision making.

In [15] the authors proposed a new method for managing the traffic system which utilizes an IoT (internet of things) concept. An intelligent traffic controller is framed with tools namely PiCamera, Raspberry Pi, IR sensors and Radio Frequency Identification. Raspberry $\mathrm{Pi}$ is the major tool which is employed to manage entire acts like a controller. The traffic density will be determined with the support of IR sensors and in order to provide zero traffic for emergency vehicles radio frequency identification technique is used. This study propose two modes of system control that is manual with introversion of human and automatic without any introversion of human.

In [16] the authors developed a smart traffic management system for control of traffic using automated electronic and mechanical devices. In the present smart city context particularly in the market and industrial zones the scenario of traffic is congested every time specifically at business hours peak time. Due to increasing development of vehicles and population in metropolitan and smart cities people faces huge issues at the main traffic points of business. It not only affects the delays in travelling but also contributes to pollution in environment as well as health risks due to pollution caused by fuels in vehicles. To resolve such problems several radiant urban communities are implementing smart control structures that run on traffic automation standards with hindrance of previously mentioned problems. In this research an enhanced traffic monitoring and control structure has been suggested that runs rapid transmission of information and their corresponding action. The simulation is undertaken using NS2 simulator which motivates the outputs in terms of good performance to manage the delay and hinder any accident due to profound congestion to a large extent.

In [17] the authors developed an internet of things based controller of traffic light in smart city. Nowadays the congestion of traffic has become a major issue in rapidly developing Indian cities which develops the air pollution. The vehicular density as well as consumption of fluid and this necessitates a new way for managing the traffic system. This has been handled through real time traffic density management using internet of things/ an intelligent traffic management system has been designed to manage the traffic system which involves components comprising of IR sensor, Raspberry Pi and display of LCD. Raspberry Pi is the major tool which is employed to manage the entire multitasking performance and IR sensor is employed to supervise the traffic density. The corresponding information is made feasible on site to show the status of traffic so that people will get early update and avoids traffic jams.

\section{Design of the System}

The study intends to propose, develop and install the Over Speeding Detector device for vehicle with the use of Internet of Things. The vehicles are fitted with over speeding detector device which has the capability for sharing information, recording and storing the data about the speed of vehicle. The following diagram shows the system architecture:

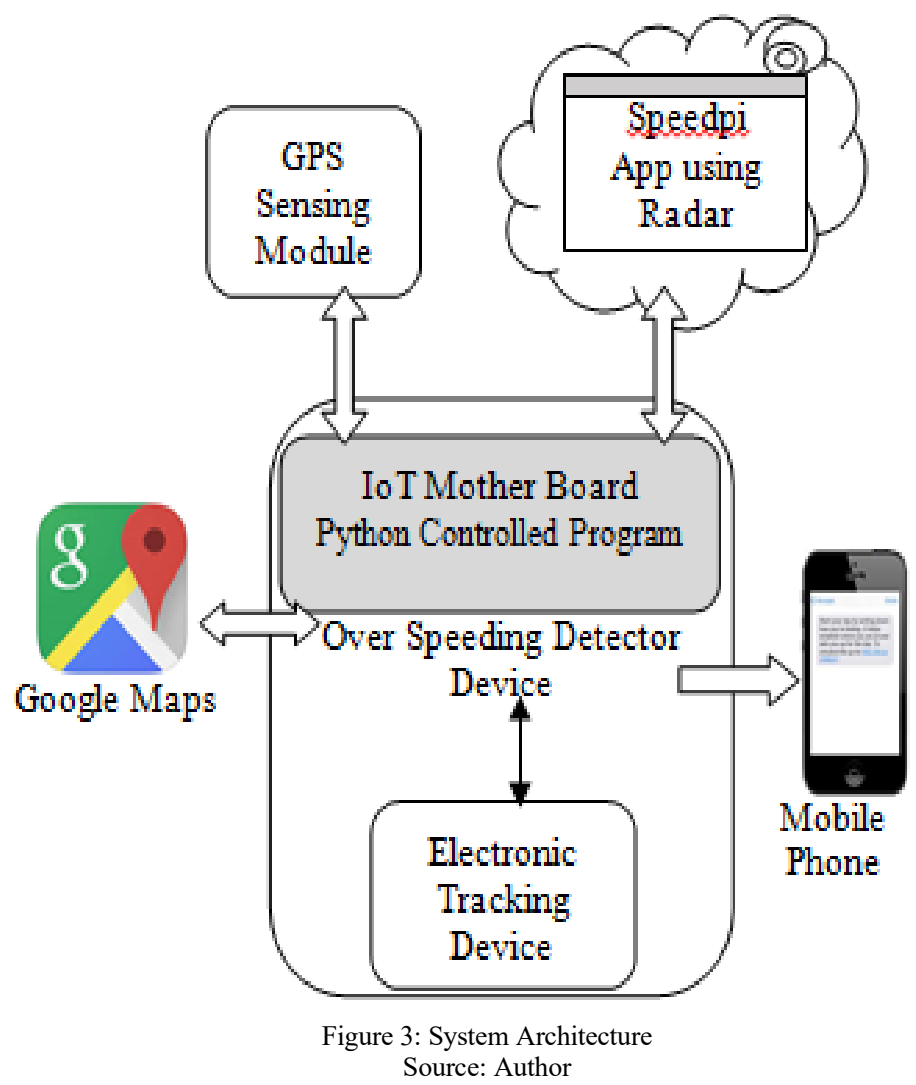

Google Maps:

Source: Author

The Google Maps Road Apps permits to plot GPS coordinates to geometry of the road and also to identify the vehicle's speed limits on the road segments. The following are services exposed for Google Maps Apps:

i. Snap to Roads: It returns the best-fit geometry of the road for a provided GPS coordinates set.

ii. Nearest Roads: It returns individual road divisions for a provided GPS coordinates set.

iii. Speed Limits: It returns the positioned speed limit for the road segment. 
GPS Sensing Module:

GPS is a constellation of 24 or more satellites flying $20,350 \mathrm{~km}$ above the surface of the Earth. Each one circles the planet twice a day in one of six orbits to provide continuous, worldwide coverage.

GPS Working

i. GPS satellites broadcast radio signals providing their locations, status, and precise time (t1) from on-board atomic clocks.

ii. The GPS radio signals travel through space at the speed of light (c), more than 299,792 km/second.

iii. A GPS device receives the radio signals, noting their exact time of arrival (t2), and uses these to calculate its distance from each satellite in view.

To calculate its distance from a satellite, a GPS device applies this formula to the satellite's signal: distance $=$ rate $\mathrm{x}$ time, where rate is (c) and time is how long the signal traveled through space.

The signal's travel time is the difference between the time broadcast by the satellite (t1) and the time the signal is received (t2).

iv. Once a GPS device knows its distance from at least four satellites, it can use geometry to determine its location on Earth in three dimensions.

Some GPS applications have the option to set speed limit warning. Generally, the longer the journey and the more measurements that are taken result in a more accurate, overall speed reading.

While some may argue that the speed returned by a GPS receiver is higher than the speedometer in the vehicle, most modern GPS receivers can collect speedometer readings using the OBDII port. This provides a method of verifying the vehicle's speed.

The GPS receiver, fitted with a cellular modem, transmits data to Speed limiter mother board by internal connection normally every minute however it can be more frequent if required. The data is processed on the Speed limiter motherboard.

\section{Working of the Over Speed Detecting Device}

The system works to determine if a vehicle is exceeding the speed limit based solely on the vehicle's speed? That's where the magic of big data comes in.

The GPS sends the speed calculated based on the aforesaid formula and this speed is send to a program inside the mother board.

The mother board actually works in two parts one a web server and second a continuously running program to monitor the incoming data from the GPS.

The Web Server contains a self-hosted web page which has a simple user interface for input. The input are

i. Mobile number for which the high speed alter should be sent.

ii. Speed Limit in $\mathrm{km}$ per hour (kmph) as integer input this will be the check value for the speed limiter.

iii. A save button will be displayed

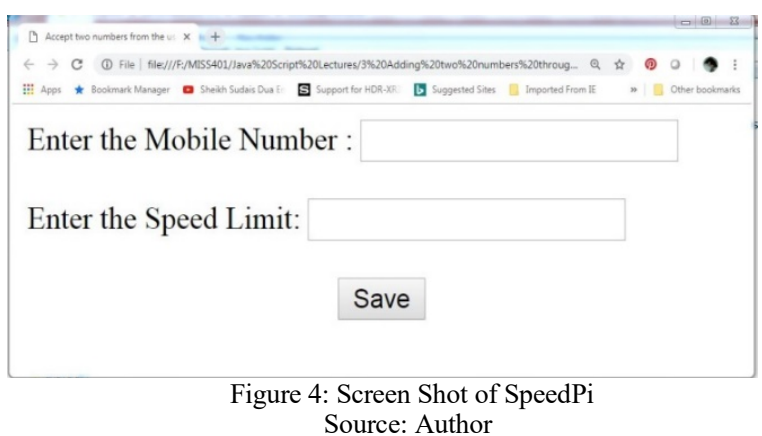

The monitor program performs seven actions,

i. Watch for the Speed data from the GPS.

ii. Based on circumference of the wheel calculate the Revolutions per second and convert it to speed. This data can be get from the OBD port if available.

iii. Cross validate the GPS speed data and calculated data.

iv. Check the GPS speed data against the speed limit value entered in the web application.

v. If GPS speed limit crosses the speed limit data then, the program activates another small routine / method which triggers the cellular modem board present alongside the speed limiter motherboard and sends out an SMS to the mobile number entered and stored via web application.

vi. The application continuously send SMS till the speed limit gets below the speed limit mentioned via application.

vii. Once the speed goes below speed limit then the application stops sending SMS and once again starts monitoring the GPS data.

The self-hosted web application can be accessed from any mobile or PC browser.

The speed limiter itself works as a $\mathrm{Wi}-\mathrm{Fi}$ access point. The mobile or PC can be connected to the speed limiter Wi-Fi network. Then open any browser and type the provided URI in instruction set above. You can see the web application, enter the relevant data and press save.

Then the application captures the data and saves it in a file inside the speed limiter mother board.

The monitor application uses the data stored as file to validate the speed limit as well as mobile number to send SMS.

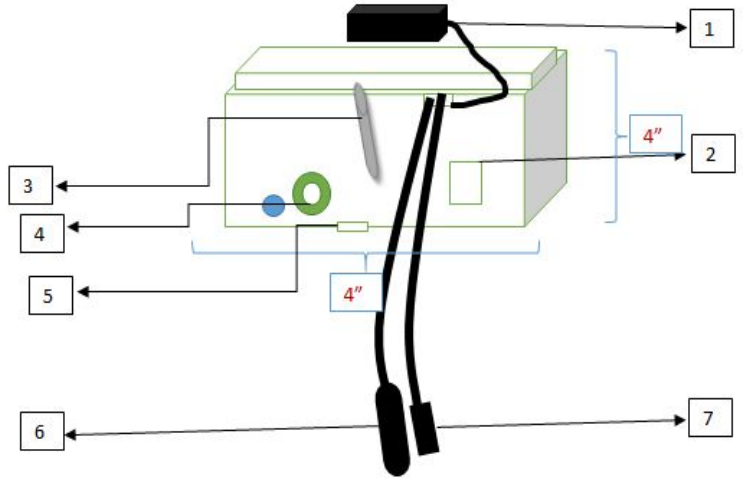

Figure 5: Device Block Diagram Source: Author 
M.A. Khan et al. / Advances in Science, Technology and Engineering Systems Journal Vol. 4, No. 2, $170-175$ (2019)

\section{Installation and Implementation}

1 - GPS receiver

3 - Mobile Unit Antenna

5 - SIM slot

7 - 05 volts 2 amp DC power input slot

The implementation steps used in detecting the over speed of smart vehicle with the use of IoT is described below with the results:

Step 1: The first step is to insert a valid SIM card with optimal cash balance of SMS in the provided slot.

Step 2: The GPS antenna is placed (a black square concealed piece) in the automobile dashboard or stick it to bonnet (hoop) outside or top of the automobile, it can be affixed to metal because of internal magnet.

Step 3: Power up the Processing Unit and GPS unit with respective 5 volts 2 amperes and 12 volts 2 amperes from the automobile charging unit or utilizing battery with enough amperage.

Step 4: Make a long press for 2 to 3 seconds after powering up the Green button. Item no 4 in reference diagram. This is too difficult and reset the mobile unit.

Step 5: Wait for 4 to 5 seconds to acquire the mobile phone tower and satellite for fixing.

Step 6: A blue button will flash continuously for every two seconds, when viewed and the blue light and one red light can be seen blinking continuously. This mean everything is better to go.

Step 7: Now on the wireless connection a mobile phone is taken and browse for mobile application named SpeedPi, choose it and enter the Raspberry password.

Step 8: Now open the URL in the cell phone and type http://192.168.0.10:8080/Index where a web page can be viewed and the relevant details are entered into it. Care must be taken that they must enter only integers in alert cell number and speed limit any space or characters which will hinder application from performing properly. Then the details are entered and press save a message named OK will be shown.

Step 9: Start the vehicle and enjoy the ride, whenever the limit of speed is crossed an SMS will be send to the cell number entered through the mobile application i.e. SpeedPi

\section{Troubleshooting and Maintenance}

i. Check the power connections for appropriate amperes using a multi meter.

ii. After finishing step 5 see inside through the spaced provided.

\begin{tabular}{|l|l|}
\hline No red light & Check the power \\
\hline $\begin{array}{l}\text { Blinking blue light with } \\
\text { blinking green light and } \\
\text { static red light }\end{array}$ & $\begin{array}{l}\text { Power off and on the } \\
\text { instrument and long press the } \\
\text { green button for two to three } \\
\text { seconds }\end{array}$ \\
\hline No blinking blue & $\begin{array}{l}\text { Satellite is not fixed place the } \\
\text { instrument in open air }\end{array}$ \\
\hline $\begin{array}{l}\text { Two blinking blue lights } \\
\text { one static red light }\end{array}$ & Normal works perfect \\
\hline
\end{tabular}

iii. SMS is not going

a. Check SIM is correctly inserted in the slot

b. Check for SMS balance

c. Check for validity of SIM card

d. Redo step two (ii) of troubleshooting.

e. Check for the proper number entered through application

f. Check for the valid speed limit entered through application

iv. Application is not opening in mobile.

a. Check for Wi-Fi connection restart the instrument and wait for few seconds and check the availability of Access point SpeedPi

b. Remember to enter proper password Raspberry

c. Ping IP 192.168.0.10 and check for reply.

\section{Conclusion}

Road accident is a major cause of accidental deaths. Among all the causes of road accidents, over speeding is leading the trail. Several efforts have been made to provide well organized and consistent ways of check and safely handle over speeding. There are many existing approaches but at times they are ineffective. The strategy for this research is to produce a much easily operable and reliable device. Since Over Speeding Detector device is based on radio frequency, the data related to the vehicle is obtained and hence tracking will become easier. Thus it can be concluded that Internet of Things is used in as one of the technology for decreasing the overspeed of vehicle and this technique can be established on roads in future which can be easily transferable.

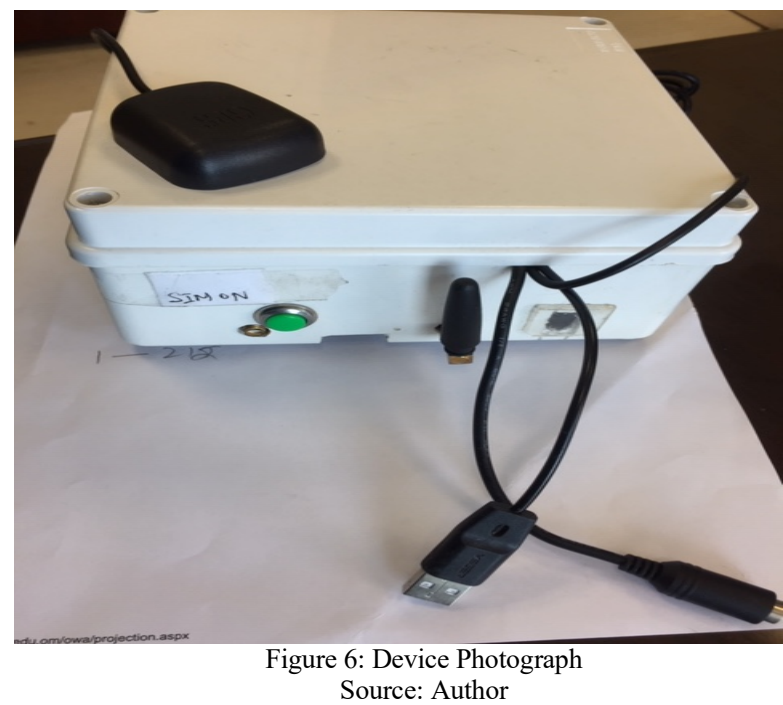

Conflict of Interest

The authors declare no conflict of interest. 


\section{Acknowledgment}

This research was funded and supported by The Research Council (TRC), Sultanate of Oman, we express our thanks and regards to them along with our serving university, Dhofar University, Salalah, Sultanate of Oman. We will also like to express our very sincere thanks to the two reviewers "anonymous", for their insights.

\section{References}

[1] Jain, M., Kumar, P., Singh, P., Arora, C. N., \& Sharma, A. (2015). Detection of Over Speeding Vehicles on Highways. International Journal of Computer Science and Mobile Computing, 4(4), 613-619.

[2] Deshpande, S., Bhole, V., Dudhade, P., Gourkar, N., \& Darade, S. (2017). Implementing a system to detect over speeding \& inform authorities in case of any violations, International Research Journal of Engineering and Technology (IRJET), Volume 04, Issue: 04, pp 2445-2449.

[3] Goenka V (2015), How Information Technology can make Indian Roads \& Highways Better and Safer!, Available at https://vinitgoenka.wordpress.com/2015/09/25/how-information-

technology-can-make-indian-roads-highways-better-and-safer/, accessed on 27th October 2018.

[4] Scroll (2018), Three killed every 10 minutes: Road accident deaths in India up $9 \%$ in 4 years, Available at https://scroll.in/article/826264/three-killedevery-10-minutes-road-accident-deaths-in-india-up-9-in-4-years, accessed on 27th October 2018.

[5] Malik Y S (2017), Road Accidents in India-2016, Available at http://www.indiaenvironmentportal.org.in/files/file/Road\%20accidents\%20i n\%20India\%202016.pdf, accessed on 27th October 2018.

[6] Thakur T T, Naik A, Vatari S and Gogate M (2016), "Real Time Traffic Management using Internet of Things" International Conference on Communication and Signal Processing, pp.6-8.

[7] Sheela. S, Shivaram. K.R, Sunil Gowda.R , Shrinidhi.L, Sahana.S and Pavithra H S (2016), "Innovative Technology for Smart Roads by Using IOT Devices" International Journal of Innovative Research in Science, Engineering and Technology,pp.1-4 Vol. 5, Special Issue 10.

[8] Chandana, K. K, Sundaram M, D'sa C, Swamy M N and Navya K (2017), A Smart Traffic Management System for Congestion Control and Warnings Using Internet of Things (IoT), Saudi Journal of Engineering and Technology, UAE,

[9] Dandala, T. T., Krishnamurthy, V., \& Alwan, R. (2017), Internet of Vehicles (IoV) for traffic management. In Computer, Communication and Signal Processing (ICCCSP), 2017 International Conference on (pp. 1-4). IEEE.

[10] Barth, M., Boriboonsomsin, K., \& Wu, G. (2013), The potential role of vehicle automation in reducing traffic-related energy and emissions. In 2nd IEEE International Conference on Connected Vehicles and Expo (ICCVE),pp. 604-605.

[11] Thakur T and Gogate M (2015), Traffic Controlling and Monitoring using Internet of Things, International Journal of Latest Trends in Engineering and Technology, Volume 6, Issue 2, pp 240-246.

[12] Bharath Kumar Perumalla and M. Sunil Babu (2015), An Intelligent Traffic and Vehcile Monitring System using Internet of Things Architecture, International Journal of Science and Research, Volume 5, Issue 11, pp 853856

[13] Lakshminarasimhan M (2016), IoT Based Traffic Management System, Available https://www.researchgate.net/publication/310036684_IoT_Based_Traffic_M anagement_System, accessed on 26th October 2018.

[14] Sonali P. Kshisagar, Priyanka H. Mantala, Gayatri D. Parjane and Kalyani G. Teke, Intelligent Traffic Management based on IoT, International Journal if Computer Application, Volume 157, Issue 2, pp 26-28

[15] Vijetha H and Nataraj K R (2017), IOT Based Intelligent Traffic Control System, International Journal for Research in Applied Science and Engineering Technology, Volume 5, Issue 5, pp 707-710.

[16] Rath M (2018), Smart Traffic Management System for Traffic Control using Automated Mechanical and Electronic Devices, IOP Publishing Private Limited.

[17] Sharma S, Giradkar V, Sanap A and Sarolkar S (2018), IOT based Traffic Light Controller in Smart City, International Journal of Advanced Research in Electrical, Electronics and Instrumentation Engineering, Volume 7, Issue 1 , pp 117-123. 